\title{
AIR COMPRESSOR SCREW PAIR PROFILING BASED ON AN ADVANCED ENVELOPE METHOD
}

\author{
LONG HOANG \\ Senior Lecturer, School of Mechanical Engineering, Hanoi University of Science and Technology, \\ Dai Co Viet, Hai Ba Trung, Hanoi, Vietnam
}

\begin{abstract}
Air compressor screw profiling is a complex problem based on the envelope theory leading to complex equations that can only be solved correctly in particular cases. To create optimized screw profiles, it needs to calculate, analyze, select, and modify variant profiles, and this can only be done with the support of the CAD systems using the advanced methods. The article focuses on the gate screw profiling from the main screw profile determined from the reverse design data, which is based on the envelope method using Boolean operation in AutoCAD. The proposed method has been implemented and verified through subroutines, written in Visual C running on AutoCAD. The testing results have confirmed that the proposed method achieves high accuracy for variant profiles in a short consumed time. This paper focuses on gate screw profiling from the main screw profile determined from the reverse design data. The air compressor screw 3D model pair, designed by using the proposed methods, have satisfied the meshing condition and was used to machine the rotors by CNC.
\end{abstract}

KEYWORDS: Profiling, Screw, Envelope \& Compressor

Received: Mar 08, 2020; Accepted: Mar 28, 2020; Published: Apr 21, 2020; Paper Id.: IJMPERDJUN20201

\section{INTRODUCTION}

The screw air compressors have been used widely since 1975 because of their high rotational speeds compared to other types of positive displacement machines, which makes them compact, their high efficiencies in a wide range of pressures and flow rates, and their long service life. The main reasons for this success is the development of novel rotor profiles, which is leading drastically reduced internal leakage and the arising of precise thread milling machine tools at approximately the same time. The advantages mentioned above is due to mainly the research achievements in the world, such as Litvin's theory on meshing condition[1] and N.Stosic's works on the compressor, etc. [2-4].

For designing the screws in practice, there are two main ways as follows: - Traditional design used for the generation of screw compressor rotor profiles is to create primary profile curves on the main screw rotor and then to generate a corresponding secondary profile curve on the gate rotor using the proper meshing condition based on the fundamental research achievements in the world. - Reverse engineering is to create 3D models screw pair by copying from a real compressor using 3D scan equipment or CMM (coordinate measuring machine). The first way achieves the high accuracy but will encounter difficulties because of the complex calculating on screw profiles satisfying the meshing condition based on the envelope theory, which cannot be solved in many cases without a numerical procedure especially when analyzing new profiles containing segments of ellipses, hyperbolae, etc.2-4 The second way seems easy but does not achieve the required accuracy so that the screw pair does not satisfy the meshing condition and then will be soon destroyed on the teeth surface. 
There is a need for new methods, that besides confronting complex conjugate shapes of the air compressor screws, will be robust in handling phenomena like undercutting, the development of the graphical design environment and its program language allows us to elaborate on new methods and dedicated software to solve the issue of study on designing air compressor screw pairs, that will be presented in this paper.

\section{METHODS FOR THE COMPRESSOR SCREW PROFILING}

\subsection{The Traditional Method in Brief}

Screw machine rotors have parallel axes and a uniform lead, and they are, therefore, a form of helical gears. As shown in Figure 1, the rotor center distance is calculated as:

$$
C=r_{1} w+r_{2} w
$$

where $r_{1} w$ and $r_{2} w$ are the pitch circle radius of the main and gate rotor, respectively. The rotors make contact line, and the meshing criterion in the cross-section is the same as that of spur gears[4].

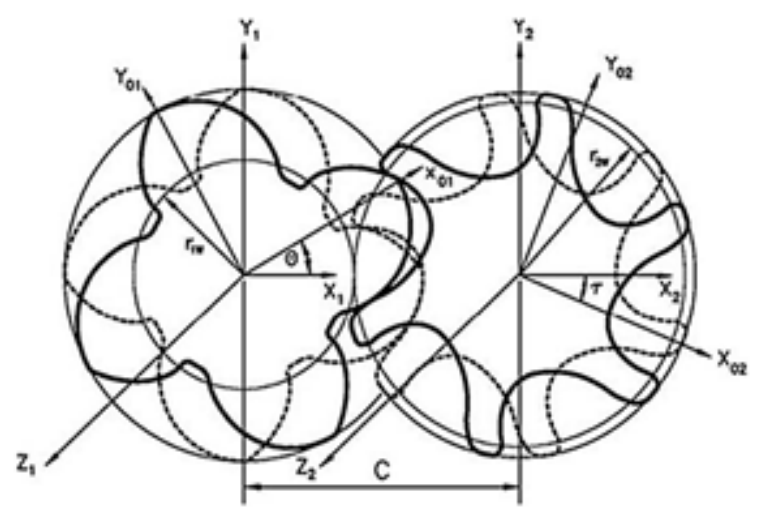

Figure 1: Screw Compressor Rotors with Parallel Shafts and their Coordinate Systems.

Starting the procedure of rotor profiling, coordinates of points on the cross-section profile of one rotor, $x O_{l}$ and y01, and their first derivatives, $d y O_{l} / d x O_{1}$, must be specified.

The problem of specifying air compressor screw profiles is solved based on the meshing condition as ${ }^{4}$ (see Figure 1a):

$$
\frac{d x o_{1}}{d y o_{1}}\left(k y o_{1}-\frac{C}{i} \sin \theta\right)+k x o_{1}+\frac{C}{i} \cos \theta=0
$$

Where $i=Z_{2} / Z_{1}=p_{2} / p_{1}, k=1-1 / i$ and $p$ is the rotor lead given for unit rotor rotation angle. Indices 1 and 2 relate to the main and gate rotor respectively. $Z_{1}, Z_{2}$ are the numbers of lobes on the main and gate rotor respectively.

Equation (2) can be solved only numerically. $\theta$ can be specified only numerically. Once obtained, the set of $\theta$ along the profile is used to calculate the meshing rotor profile point coordinates.

Since $\tau=\theta / i$, the meshing profile equations of the gate rotor in the cross-section are calculated as [4]:

$$
\begin{aligned}
& x o_{2}=x o_{1} \cos k \theta-y o_{1} \sin k \theta-C \cos \theta / i \\
& y o_{2}=x o_{1} \sin k \theta+y o_{1} \cos k \theta+C \sin \theta / i
\end{aligned}
$$




\subsection{The Proposed Gate Screw Profiling Method}

\subsubsection{Reverse Engineering Data}
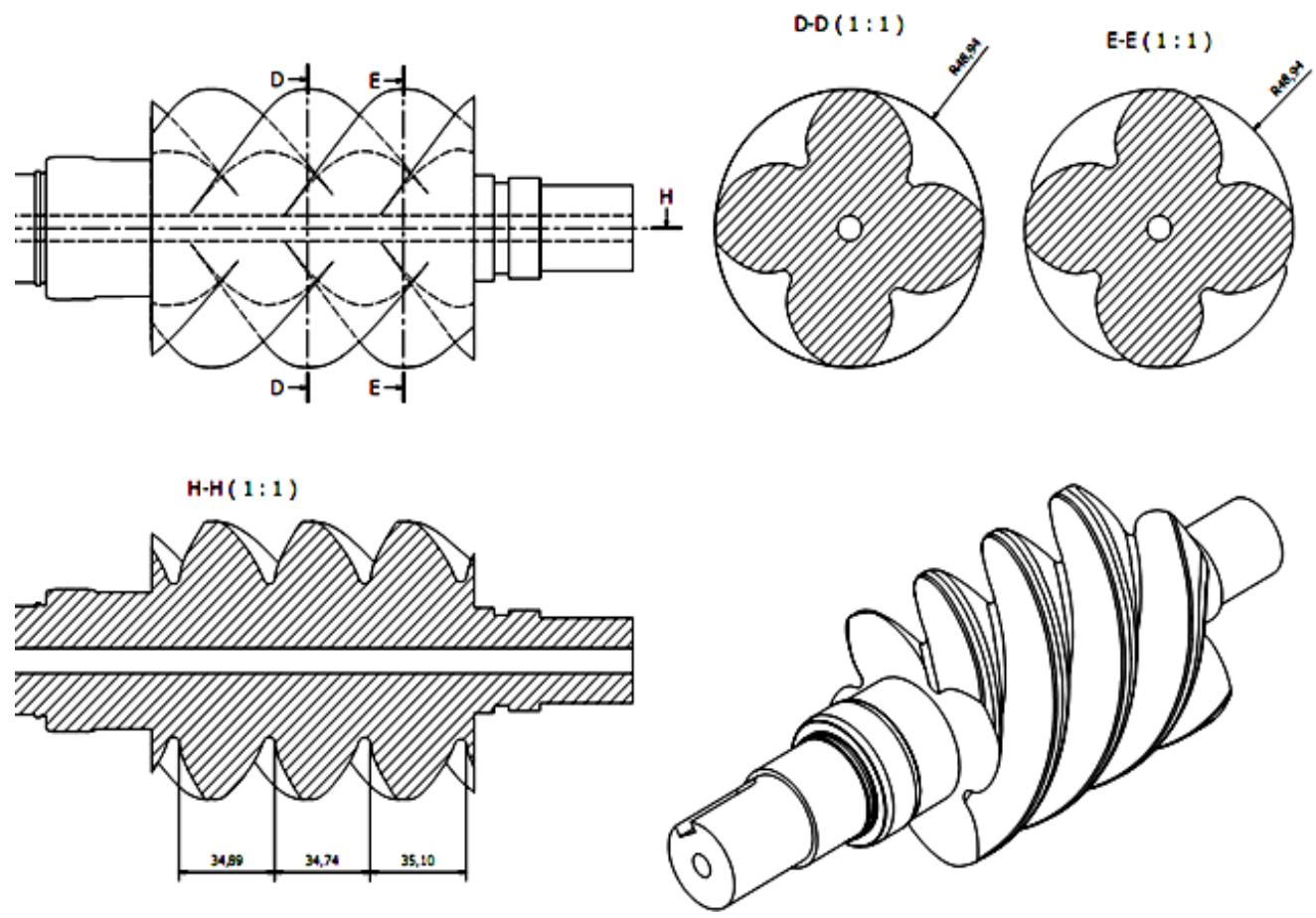

Figure 2: The Main Screw 3D Model created by Reverse Engineering.

After using reverse engineering, the 3D model of the main screw was not accurate, as shown in Figure 2 and Figure 3(a), so it needs to correct the profile on a cross-section of the main screw and then to create the gate screw profile from the correct profile of the main screw as below.

\subsubsection{Correcting the Main Screw Profile}

From the reverse engineering data, the profile on a cross-section of the main screw was analyzed into the segments, as shown in Figure 3(b). Where BC is an Epicycloid, that has generated by a point lying on a circle of radius $35 \mathrm{~mm}$, called an epicycle. The epicycle is rolling without slipping around the pitch circle of radius $30 \mathrm{~mm} . \mathrm{CD}, \mathrm{DE}, \mathrm{EF}, \mathrm{FG}, \mathrm{GH}$ are circles, and they are tangent each to the next one. The center point of DE is on the axis of the main screw. AB and HI are ellipse segments.

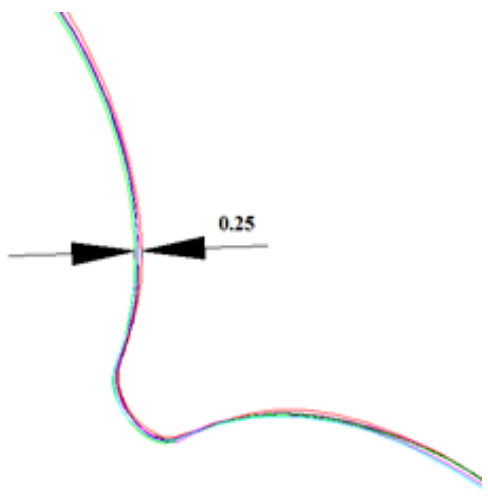

(a)

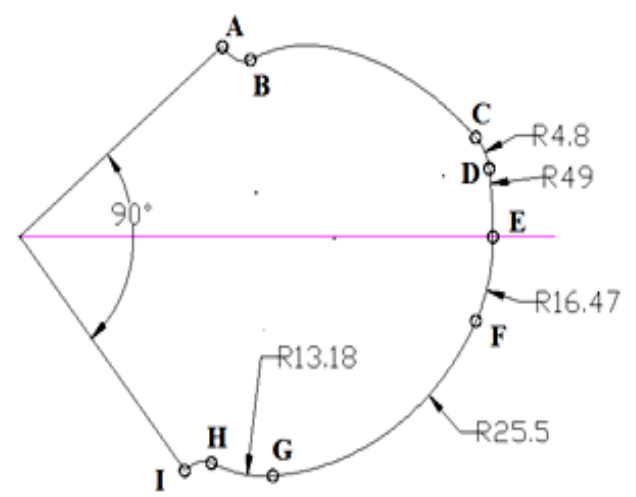

(b)

Figure 3: (a) Difference of the Main Screw Profiles; (b) Correcting Main Screw Profile. 


\subsubsection{The Algorithm for Automatic Creating the Gate Rotor Profile}
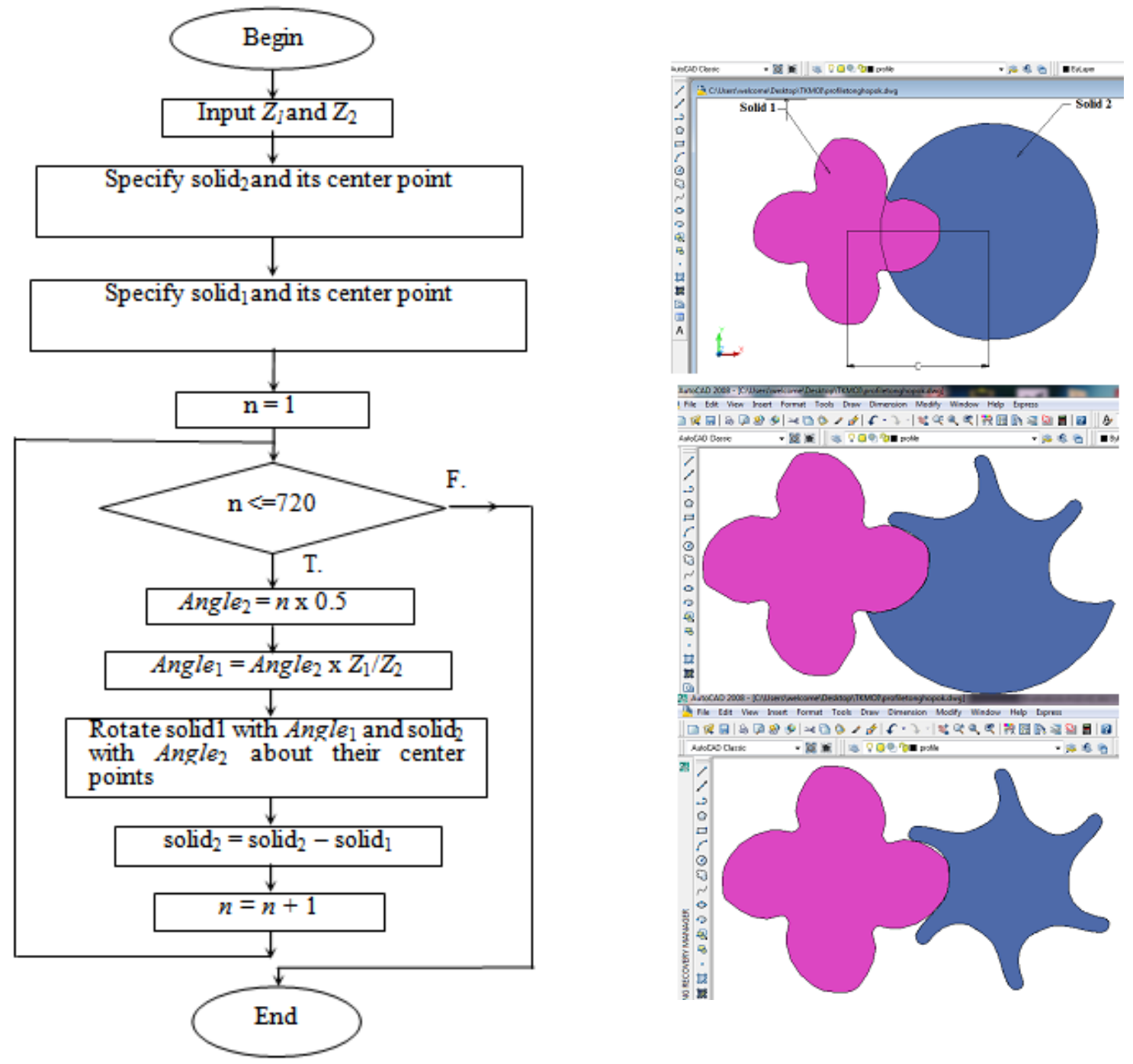

Figure 4: (a) The Algorithm for Creating the Gate Screw Profile; (b) The Gate Screw Profile was being Created.

The algorithm for generating a gate rotor profile from the main rotor profile is shown in figure 4(a). Where Solid 1 is created from the main rotor profile (in section 2.2.2), Solid 2 is created at first from a circle, Solid 1 and Solid 2 were placed with the distance $C$ calculated by Eq. (1). Solid 2 will become a gate rotor profile after performing the above algorithm (see Figure 4b). The statement, Solid2 - Solid 1, is achieved by using the "Subtract" command in AutoCAD. The numbers, 720 and 0.5 , correspond with a turn of the main rotor.

\section{VERIFICATION RESULTS AND DISCUSSIONS}

The algorithm provided in section 2.2.3 has been implemented into the AutoCAD environment. The subroutine written in AutoLisp, running in AutoCAD, produced the gate screw profile, as shown in Figure 4(b).

For verification of the method, the gate rotor profile created from the main rotor profile used again as the input, the main rotor profile was created by the same method as the method creating the gate rotor profile. Using Geomagic Control X, compare of two main rotor profiles was shown in figure 5(b). The gate rotor profile created from the main rotor profile by using the proposed method was also placed with overlapping the gate rotor profile determined from the reverse 
engineering data of the real gate rotor, as shown in figure 5(a). The maximum difference between the two profiles is 0.21 $\mathrm{mm}$ at their crests. In other areas, the differences are less than $0.02 \mathrm{~mm}$.

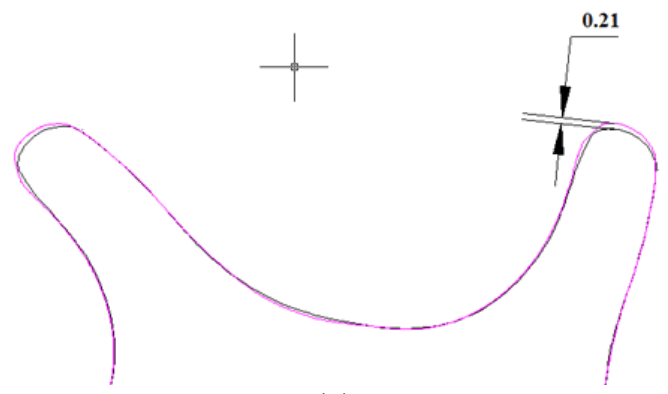

(a)

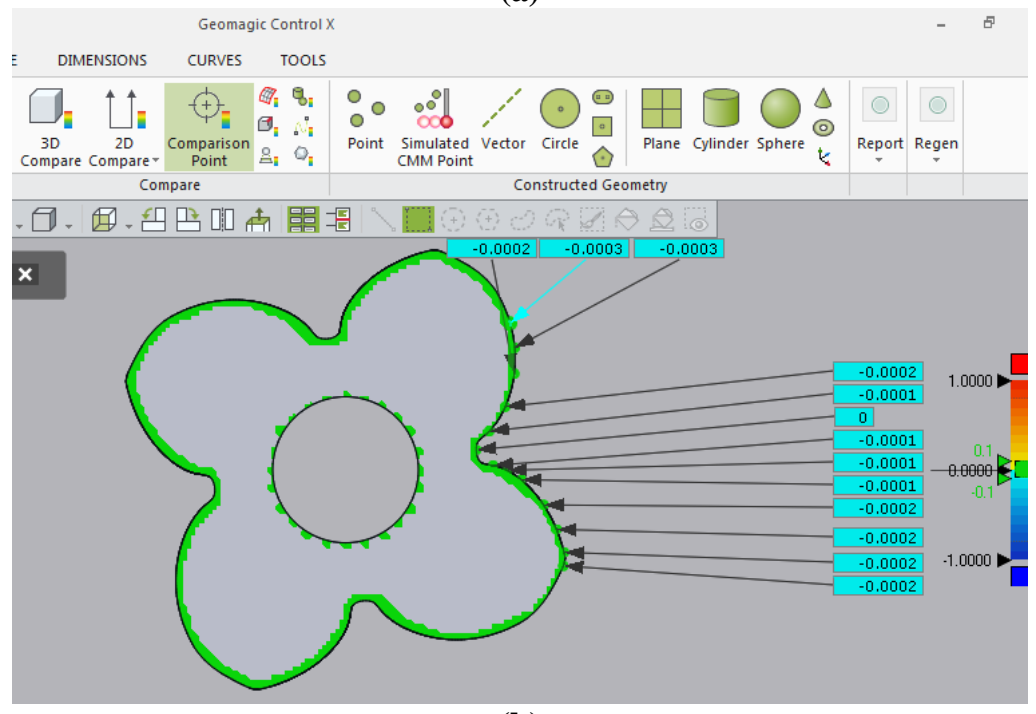

(b)

Figure 5: (a)Compare Two Gate Rotor Profiles; (b) Accuracy Verification of the Proposed Method.

Form the two profiles created above, using the "SWEEP" command in Auto CAD, create 3D solid models of two screws with the constant leads specified from reverse engineering data, as shown in Figure 6(a). The 3D solid models of two screws were used to machine by $\mathrm{CNC}$, and the machined screw pair is shown in Figure 6(b). The machining accuracy of the screw pair is shown in Figure 7.

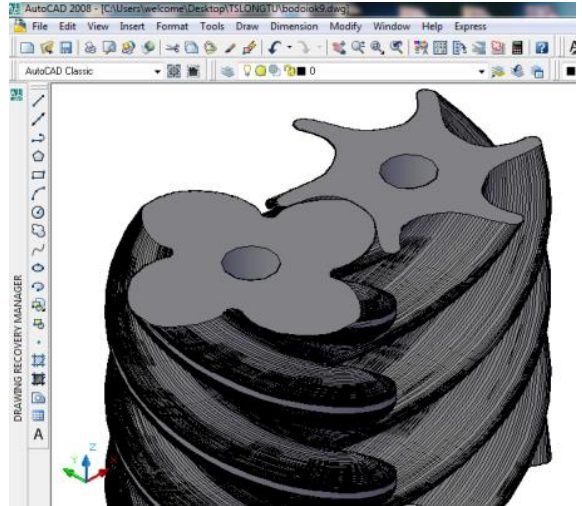

(a)

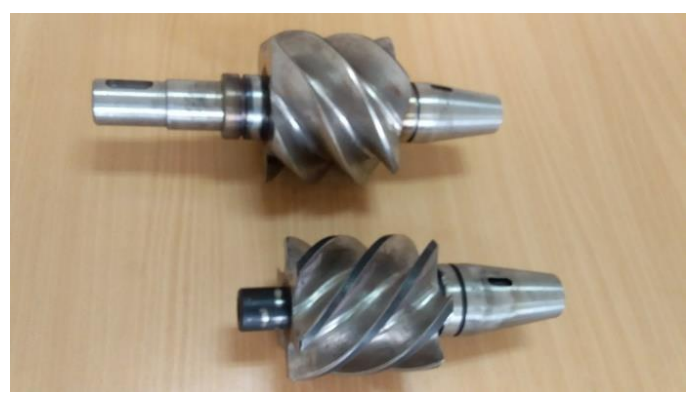

(b)

Figure 6: (a) 3D Solid Model of the Screw Pair; (b) The Screw Pair after Machining by CNC. 


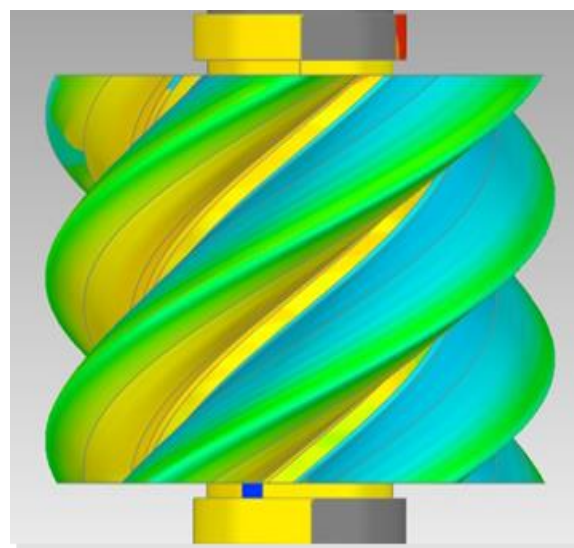

3D Deviation

Max + / : $0.1000 /-0.1000 \mathrm{~mm}$

Average + /-: $0.0492 /-0.0498 \mathrm{~mm}$

Standard Deviation: $0.0569 \mathrm{~mm}$

RMS Estimate: $0.0571 \mathrm{~mm}$

Measure CSYS: World CSYS

View CSYS: World CSYS
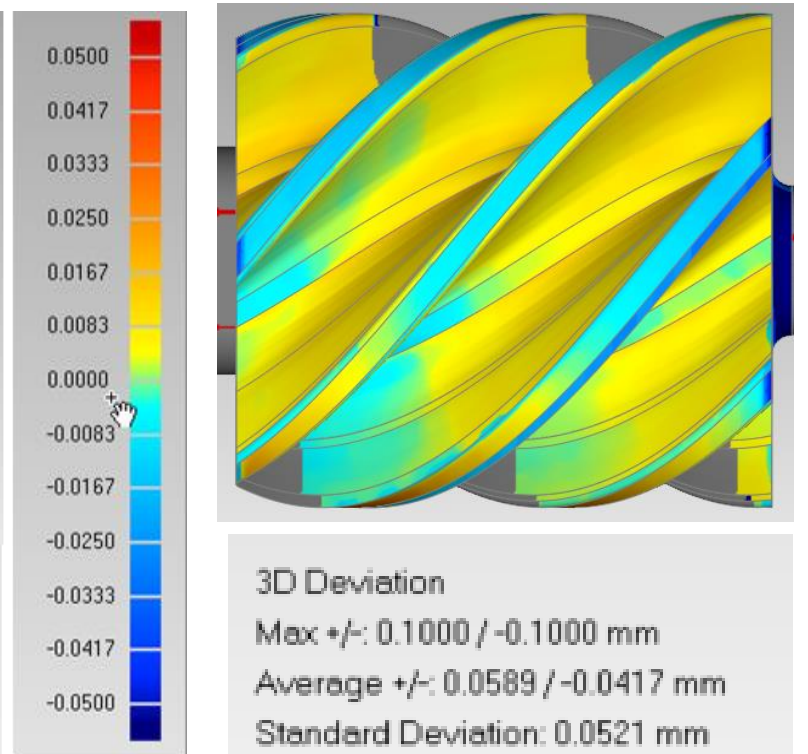

3D Deviation

Max +/: $0.1000 /-0.1000 \mathrm{~mm}$

Average $+/=0.0589 /-0.0417 \mathrm{~mm}$

Standard Deviation: $0.0521 \mathrm{~mm}$

RMS Estimate: $0.0621 \mathrm{~mm}$

Measure CSYS: World CSYS

View CSYS: World CSYS

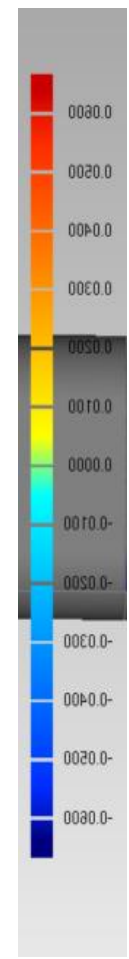

Figure 7: 3D Comparison between the 3D CAD Model and the Machined Screws

Figure 5(b) has shown that the proposed method creating a screw profile from the given corresponding screw profile is absolutely accurate $(<0.003 \mathrm{~mm})$ but figure $5(\mathrm{a})$ has shown that the maximum difference between the two profiles is $0.21 \mathrm{~mm}$ at their crests, that is due to reasons such as:

The main and gate rotor profiles determined from the reverse engineering data are not accurate, this reason can be found from the figure $3(\mathrm{a})$;

The main and gate rotor profiles were smaller after working in a long time;

The clearance between the crest of the gate rotor and the root of the main rotor is the need for the oil-injected $[5,6]$.

In spite of the difference between the two rotor profiles as shown in figure 5(a), that has been explained above, from the figure 5(b), it may state that the meshing condition of two rotors seem satisfied absolutely so that the screw pair created as above must be better than the screw pair created by reverse engineering that consumes a long time and has low accuracy (see figure 2 and 3a). From the testing results and analysis above, it may be sure that: The proposed method used for creating the gate rotor profile from the main rotor profile has achieved high accuracy and is general for any given rotor profile. Applying the proposed method, we can save design time, finance for reverse engineering, and, more importantly, improve the quality of the air compressor screws.

\section{CONCLUSIONS}

The proposed method for profiling air screw pair achieves high accuracy, is suitable for given variable profiles such as the profile created by reverse engineering or new profiles. The proposed method is easy to apply, as well as handles phenomena like undercutting or singular point, and will be the foundation to study new air compressor screw profiles as well as to reproduce damaged screw pairs. 


\section{ACKNOWLEDGMENTS}

This work was supported by Hanoi University of Science and Technology in the project T2016-PC-077.

\section{REFERENCES}

1. Litvin F.L, Development of Gear Technology and Theory of Gearing, NASA RP 1406 (1994).

2. Hanjalic K, Stosic N, Development and Optimization of Screw Machines with a Simulation Model, Part II: Thermodynamic Performance Simulation and Design, Journal of Fluids Engineering, 119 (1997) 664-671.

3. Stosic N, Hanjalic K, Development and Optimization of Screw Machines with a Simulation Model, Journal of Fluids Engineering, 119 (1997) 659-667.

4. Stosic N., On Gearing of Helical Screw Compressor Rotors, Journal of Mechanical Engineering Science, 212 (1998) $587-595$.

5. Fujiwara M, Osada Y, Performance Analysis of Oil Injected Screw Compressors and their Application, Int J Refrig, 18 (1995) 4-12.

6. Sauls J, The Influence of Leakage on the Performance of Refrigerant Screw Compressors, Proc. VDI Tagung "Schraubenmaschinen 94", Dortmund VDI Berichte 1135 (1994).

7. P. Ravinder Reddy \& M. Saikiran, " Aerodynamic Analysis of Return Channel Vanes in Centrifugal Compressors ", International Journal of Mechanical Engineering (IJME), Vol. 5, Issue 1, pp. 73-82

8. Bhavinkumar N Patel \& Dattatraya Subhedar, " Experimental and Numerical Investigation of Diesel Engine Turbocharger ", International Journal of Automobile Engineering Research and Development (IJAuERD), Vol. 6, Issue 3, pp. 11 - 16 
